\title{
Diagnostic pathways in Crohn's Disease
}

\section{Introduction}

Crohn's disease (CD) is a chronic, relapsing and remitting, transmural inflammatory disease of the gastrointestinal (GI) tract of unknown aetiology (1). Historically, the roles of imaging in CD have been to aid diagnosis (particularly for endoscopically-inaccessible segments) and identify complications. However, as CD treatment has evolved, imaging has assumed a central role in activity assessment, treatment monitoring and, increasingly, predicting patient trajectory.

Traditionally, CD management involved stepwise escalation of corticosteroids, immunomodulators, and biological anti-tumour necrosis factor (TNF) agents (2), aiming to control symptoms. However, this approach may delay initiation of the most effective therapies, resulting in poorer outcomes due to uncontrolled subclinical inflammation $(3,4)$. It is insufficient to rely on patient symptoms to guide treatment intensity, because such symptoms rarely reflect inflammatory burden or presence of irreversible bowel damage. Therefore, objective tests are needed, with treatment being escalated until such tests normalise (or at least improve). In support of this "treat-to-target" concept, the recent CALM randomised trial showed that CD treatment titrated to blood and stool biomarkers (C-reactive protein and calprotectin) resulted in superior rates of bowel healing at $1 \mathrm{yr}$ than treatment guided by symptoms alone (5). The implications for imaging are obvious - we must not only be able to diagnose CD and its distribution, but depict and quantify the degree of inflammatory activity and response to treatment, permitting adjustment if necessary.

In this review, we summarise the principal imaging modalities available for $C D$ imaging, key findings, and provide guidance on imaging options at various points in the patient pathway, focusing on clinical decision-making at each step. Alternative reviews and 
guidelines are available for discussion of the technical characteristics of individual imaging tests $(2,6)$. We then offer a brief discussion of current developments in CD imaging, and practical considerations regarding service configuration and delivery.

\section{Principal Imaging Modalities}

\section{Barium Follow-Through (BaFT)}

Historically, BaFT was the workhorse for both suspected and confirmed CD, identified as the most commonly-performed investigation for luminal small bowel CD in a 2011 survey(7). Since then, use has dwindled by 15-20\% year-on-year in England (8), with fewer than 6000 examinations per annum currently. It imparts ionising radiation and has inferior diagnostic yield to modern cross-sectional modalities (9). Skills in competent acquisition and interpretation have atrophied, meaning it is now reserved for specific problem-solving such as assessment for adhesions, strictures or where enteric transit time is questioned and alternatives are unavailable.

\section{CT enterography (CTE)}

Enterography involves drinking oral contrast, whereas enteroclysis describes instillation via a nasojejunal tube. Collapsed bowel simulates or masks disease, necessitating luminal distension (10-12). Contrast can be neutral (similar attenuation to water; usually polyethylene glyocol or mannitol) or positive (higher attenuation than water; iodine or barium). Hyperosmolar neutral contrast allows assessment of bowel wall thickness and enhancement without compromising mesenteric assessment (13). Positive contrast differentiates bowel from extraluminal fluid, inflammatory changes and lymph nodes, although evaluation of bowel wall enhancement is compromised (14); it is therefore most useful for detecting sinuses and fistulas $(15,16)$. All modern CT scanners are capable of the thin-section (<2mm), rapid imaging needed for CTE, and pump injectors permit rapid 
injection (3-4 mls/s) of $100-120 \mathrm{mls}$ of iodinated contrast at $45-60$ second delay.

Antispasmodics such as hyoscine butylbromide minimise motion and peristaltic activity. The major downside of CTE is radiation dose; even with modern scanners, 3-5 mSv is commonplace. As a one-off test, this is of limited importance, but where the test must be repeated, as may occur in IBD, dose (and risk) may accumulate rapidly - reaching $75 \mathrm{mSv}$ (and $a>7 \%$ increased cancer risk) in over $15 \%$ of patients in one audit (17). International guidelines (18) acknowledge that BaFT and CTE are no more sensitive than alternatives that do not use ionising radiation, and so should be avoided where possible.

\section{Small Bowel Ultrasound (SBUS)}

SBUS is readily available, inexpensive, non-invasive, well-tolerated and confers no radiation exposure (19). It provides comprehensive evaluation of both the bowel and the solid abdominal viscera. A fasting period of $>4$ hours and high frequency probes $(5-17 \mathrm{MHz})$ are recommended (20). In most cases, image quality is excellent, particularly with newer scanners; but may be less reliable in obese patients. It is of particular value in paediatric and adolescent populations, who are generally slimmer and tolerate it well. Oral and intravenous contrast can be added, but are not routine in most centres. Although often described as highly operator-dependent, with training and practice this becomes a lesser issue; interreader agreement for key parameters at US (21) is comparable to endoscopy (22) or MRE (23).

\section{MR enterography (MRE)}

As for CTE, MRE involves drinking $1-2 \mathrm{~L}$ of oral contrast, commonly $2.5 \%$ mannitol, over 40-60 minutes before the scan, although some advocate smaller volumes with alternative agents such as lactulose. The cornerstone of imaging is rapid T2-weighted sequences, with and without fat suppression. These are supplemented by one or more of contrast-enhanced 
T1-weighted, diffusion-weighted and cine motility sequences (Table 1). Antispasmodics are mandatory, and field strengths of $1.5 \mathrm{~T}$ or greater are ideal, although claustrophobic patients can sometimes be imaged successfully at lower field (0.5 to 1.2T) on an open magnet. Since MRE confers no ionising radiation and generates highly reproducible images, it is ideal for serial assessments. However, it is expensive, time-consuming, and less well-tolerated than SBUS (24). Its manifest advantages mean that, currently, >35,000 MRE examinations are performed each year in England alone (8).

\section{The Roles of Imaging}

\section{Diagnosis and initial disease phenotyping}

CD ranges in severity from minimal inflammation, requiring no treatment, to lifelong refractory disease. This spectrum implies that CD, like cancer, must be "staged" following diagnosis to determine the choice and intensity of treatment. Isolated ileocolonic CD, for example, may be well-suited to surgical management, particularly with obstructive symptoms. Conversely, inflamed proximal "skip lesions" may mandate a pharmacological approach, at least initially. Accordingly, international consensus (18) recommends that imaging be performed at diagnosis. When confronted with a patient in whom CD is suspected, there are two related but conceptually separate questions - firstly, does this patient have CD; and, secondly, what is its location and severity? Much of the literature deals with the first question, but not the second.

Despite the interest in imaging diagnosis of $C D$, there are few head-to-head comparisons of imaging used in the same patient cohort. For example, several metaanalyses reported that MRE, US, CTE and BaFT have similar diagnostic sensivity for CD $(25,26)$, but these mainly include single-centre studies in which patients underwent one test or another, comparing each against a different standard of reference (e.g. endoscopy or surgery). So, these data show the average pooled sensitivity of the different tests when 
employed in a variety of different clinical settings. This does not permit unbiased comparison of the tests themselves, as they were used in different patients. The recently-published METRIC study directly addressed this by comparing MRE and US in both suspected and established CD in a prospective, multicentre UK setting (27). All patients underwent both tests, with the operators (representative UK radiologists) blinded to other clinical, radiological and endoscopic information. A consensus panel used all available data after 6 months follow-up to determine the presence, site and activity of disease, forming a robust "gold standard" against which to judge MRE and SBUS. This showed that both tests were highly accurate for the presence of small bowel CD, but MRE was superior. MRE had sensitivity of 97\% whereas US was $92 \%$ sensitive, a small but statistically-significant difference. MRE was also more specific (96\% vs $84 \%$ ), a non-significant difference. When considering both disease presence and its segmental location and activity, MRE was both more sensitive and specific than US (MRE: sensitivity $80 \%$, specificity $95 \%$; US: sensitivity $70 \%$, specificity $81 \%$ ) (Figure 1).

These findings have important implications for routine clinical practice. Because it is highly sensitive for disease presence at 92\% (albeit lower than MRE), US could be used as a first-line investigation in those considered at low risk for $C D$. This may be in conjunction with faecal calprotectin, which is highly sensitive for inflammation but relatively non-specific, and probably less effective for Crohn's disease than colitis(28), particularly if proximal disease; it is also difficult to interpret if patients have a stoma. If both tests are normal, active IBD is essentially excluded. Conversely, once a diagnosis of CD is established, in most hands MRE is superior for disease mapping (i.e. establishing location and activity), and can be employed at this stage. However, this may not be the case at all centres and for all patients; for example, in children and adolescents, there is excellent agreement between US and MRE(29). 


\section{Key Imaging Findings at Diagnosis}

At first presentation of $C D$, many patients have imaging findings that overlap with other conditions. This may lead to the radiologist issuing a generic report; "this may be inflammatory, infectious or ischaemic", and advising the dreaded "clinical correlation". Yet there are nuances which may permit greater specificity.

Findings at diagnosis depend heavily on (a) the degree of activity (i.e. inflammatory burden), (b) the presence of disease chronicity and bowel damage and (c) penetrating and/or stricturing complications. Where the disease is very mild, and without gut damage or complications, imaging may be normal (or near-normal). Although barium studies occasionally depict superficial disease as aphthous ulceration, in reality the test is relatively insensitive(30). Wall thickening on cross-sectional imaging (typically using $3 \mathrm{~mm}$ as the cutoff) is an early sign of enteric abnormality, but can be caused by virtually any gut disease. Similarly, presence of mural oedema (intermediate density "water halo" on CT or T2 hyperintensity on MRI) and hyperenhancement are non-specific findings, often present in infection, other inflammatory processes, or with ischaemia (particularly if venous or with reperfusion). These signs should therefore be used to detect abnormality, rather than categorise its cause.

Other ancillary features may be more discriminatory. Clinical history is often helpful; ischaemia is typically rapid onset (minutes to hours), infection slightly less rapid (hours to days) and inflammation, including IBD, generally longer still. Similarly, systemic upset and inflammatory markers are usually low-grade or grumbling in CD, unless complicated by acute bowel obstruction or an abscess. With regard to imaging features specifically, enteric distribution is of course helpful. The combination of patchy ileocolonic abnormality, centred on the ileocaecal valve, accompanied by skip proximal segments and enteric and perianal fistulas is virtually pathognomic of Crohn's; but this "full house" of textbook findings is the exception rather than the rule. CD is an asymmetrical, patchy disease in many respects - it characteristically affects the mesenteric side of the gut more than the anti-mesenteric. 
Therefore, even within the same imaging slice showing a single axial section through the bowel, there is often heterogeneity rather than the homogenous, oedematous swelling more commonly seen in infection or ischaemia (Figure 2a). Normal bowel folds are lost, and the interface between the bowel wall and its mesentery is blurred, findings that are particularly well shown on ultrasound (Figure $2 b$ ). The mesentery itself is frequently expanded, oedematous and heterogenous, with "fat-wrapping" visible as finger-like creeping expansion of the mesenteric fat towards the anti-mesenteric side (Figure 2c). As well as being patchy within the axial plane of the bowel, CD is discontinuous along its length - the classic "skip lesion". Even without a truly separate block of disease, close inspection of an apparently contiguously-involved segment almost invariably shows some degree of heterogeneity, with variations in wall and mucosal thickness, oedema (high T2 signal or mixed echogenicity on MRE and US respectively) and vascularity/enhancement (Figure 2d). This variability in both the axial and longitudinal planes, in combination with other better-known features (distribution centred on ileocaecal valve and fat wrapping) may allow the radiologist to suggest $C D$ as the most likely diagnosis.

Once the diagnosis of CD is made, it is important to provide a broad description of its phenotype and behaviour, often summarised as (a) non-stricturing, non-penetrating (inflammatory), (b) stricturing, or (c) penetrating. These three groups, although described separately in the commonly-used Montreal classification (31) as B1, B2 and B3 respectively, in reality may overlap, with parts of the bowel being mainly inflamed, other parts showing mixed inflammatory and fibrotic strictures, and co-existent fistulas. The main benefit of such terminology is in prognostication - stricturing and penetrating phenotypes are associated with poorer longer-term outcomes, often needing surgical rather than medical therapy.

\section{Activity assessment}


Robust activity assessment in CD is critical since suppression of inflammation prevents long-term complications. Imaging is intuitively attractive since (unlike colonoscopy) it evaluates the entire GI tract, including the perianal region if required; and it not only quantifies disease activity but also shows its distribution and presence of complications.

Although endoscopy is the current gold standard for activity assessment, standardised imaging criteria also depict and quantify inflammatory burden (32-34). The magnetic resonance of activity (MaRIA) score is perhaps best known, and was derived by correlating various MR features with the Crohn's Disease Endoscopic Index of severity (CDEIS) score(35). After regression modelling to determine the most useful MRI features, the MaRIA score was derived as follows: $1.5^{\star}$ bowel wall thickness $(\mathrm{mm})+0.02^{\star} \mathrm{RCE}+$ $5^{\star}$ presence of oedema $+10^{*}$ presence of ulceration. A score of $<7$ indicates no disease activity, and $<11$ indicates there will be no bowel ulceration endoscopically (i.e. only minimal activity).

Another validated scoring system is the Crohn's Disease MRE Index (CDMI), originally developed against histological activity scores (36). It uses semi-quantitative assessments of bowel and adjacent tissue signal, alongside wall thickness. Each is graded from $1-3$, and applied to the scoring equation: $1.79+1.34^{*}$ mural thickness score $+0.94^{*}$ mural T2 signal score. A score of $\geq 4.1$ represents active disease. To generate a score across the whole gut, rather than just a single segment, CDMI has been extended to include the length of abnormal bowel and the presence of extraluminal complications, generating the magnetic resonance enterography global score (MEGS), which has been validated against both calprotectin(37) and capsule endoscopy(38) (Table 2).

Although MaRIA and MEGS are reproducible between radiologists and sensitive for active $\mathrm{CD}(35,36,39)$, they are too cumbersome for everyday practice, where the key questions are simpler; firstly, is there any activity at all; and, secondly, what is its severity. The former is often straightforward; active disease is indicated by the presence of the major components of the scores mentioned above. The combination of wall thickening and mural 
oedema typifies active disease; when neither of these features are present, there is unlikely to be significant activity. Wall thickening alone, without supporting features of oedema or ulceration, often indicates inactive disease, particularly if the wall is dark on fat-suppressed T2-weighted images (Figure 3a). T2-weighted images without fat suppression are less helpful, as both mural fat (indicating inactive disease) and mural fluid (indicating active disease) will be bright (Figure 3b). Contrast enhancement may be useful, but active and fibrotic disease both enhance $(40,41)$. Early, brisk enhancement is more typical for active CD whereas delayed enhancement may indicate established fibrosis(42). However, activity and fibrosis often co-exist, so the assumption of "increased enhancement=active" is an oversimplification. Accordingly, and particularly given recent awareness of brain deposition of gadolinium with MR contrast (43), several newer activity scores give less weight to contrast enhancement or disregard it entirely (44-46).

Having established the presence of active disease, we suggest simple quantitation of its severity; mild, moderate or severe (roughly corresponding to grades 1-3 for each component of the MEGS score). It is quick and easy to assess each bowel segment, and then mentally pool these to make an overall judgement regarding global activity.

When using ultrasound, a similar principle applies; active disease must be detected and quantified. The hallmarks of active disease, wall thickening and oedema (low echogenicity), are well-shown by ultrasound (Figure 4), and can be supplemented by Doppler techniques, which are consistently associated with active disease (47-49). All of these parameters can be combined into US activity scores $(48,49)$, although, as for MRI, these are not routinely deployed in clinical practice, and the simpler description of mild, moderate and severe is sufficient.

\section{Therapeutic monitoring}

CD treatments aim to improve symptoms and preserve bowel health and length, while minimising complications. Suppression of inflammation is a proxy for these goals. 
International consensus now recognises imaging as an appropriate target for therapy (50), with the caveat that endoscopic assessment is preferred if feasible. Frequently, however, the disease is either endoscopically-inaccessible, or regular colonoscopies are too burdensome - therefore, imaging becomes the monitoring tool of choice.

Although there are no randomised trial data confirming that treating to an imaging target (e.g. suppression of all inflammation on MRI/CT/US) leads to superior clinical outcomes (e.g. need for surgery) than therapy guided by symptoms alone, observational studies are supportive. For example, patients who achieved normalisation of cross-sectional imaging (CTE/MRE) on treatment had lower rates of hospitalisation or surgery than those who had ongoing imaging abnormality (51). If we thus accept the "treat-to-target" concept, the immediate question becomes, "what is the appropriate target?". There is broad consensus among gastroenterologists that best outcomes occur with endoscopic mucosal healing(52). By analogy, we should aim for no or minimal activity on imaging. Ongoing activity should trigger treatment adjustment; for example, initiation of biological treatment, dose escalation or a switch in therapy. On the other hand, where imaging remission has been achieved, it may be possible to cautiously reduce therapy, thereby sparing patients from their sideeffects and reducing costs.

In practical terms, it is useful to draw a distinction between response to treatment, and imaging remission. This is similar to partial response and complete response in cancer imaging, and indeed this oncologic terminology has been applied to CD (51). Response implies a significant reduction in inflammatory burden, and in drug trials using endoscopy, often requires $>50 \%$ reduction in endoscopic scores (53). Clinical practice is less rigid, particularly for imaging, but a reasonable rule-of-thumb is to use the activity categories of mild, moderate and severe described above, and regard a reduction of one category as minor response, two points as good response. Imaging remission entails abolition of all activity. Since this assessment requires direct comparison of two tests, judgement is easier when comparing like-with-like. Therefore, if possible, the same modality should be used for 
longitudinal follow-up. Moreover, when performing ultrasound, it is critical to save multiple images (or video clips) of each abnormal segment, to ensure robust comparison (Figure 5).

\section{Monitoring Gut Damage and Complications}

The next major facet of imaging in $C D$ is to monitor and categorise the very gut damage that treatment aims to prevent. Global bowel damage scores have been proposed, the best known being the specifically-developed Lemann index $(54,55)$. Each segment (upper GI tract, small bowel, colorectum and perianal region), is scored 0-3 based on the degree of stricturing, penetrating disease and previous surgery/resection. This is done using a combination of MRE (or CTE) and endoscopy; presently, it has not been validated for ultrasound. The score emphasises irreversible change/complications over inflammatory activity. Tailored management can be based on both absolute values of the index and progression over time. Presently, it is a research tool, since its calculation is both complex and time-consuming. However, the concept of monitoring cumulative bowel damage is important, and radiologists must assess and communicate this. Ideally, terminology should be standardised; the authors encourage readers to refer to recent consensus recommendations for appropriate terminology and image description in CD (56).

Development of CD-related complications is inherently linked to accumulation of bowel damage, since the former often leads to the latter. Broadly speaking, complications can be divided into stricturing, penetrating, and related to short bowel; with development of malignancy a rare but feared occurrence. Strictures (stenoses) are best demonstrated by "stressing the bowel" with an oral contrast load; when this is done (including at ultrasound),

all the major cross-sectional imaging techniques are equally effective (25). The definition of a stricture is variable, but at a minimum requires a fixed luminal narrowing; confident diagnosis requires upstream dilatation to $>3 \mathrm{~cm}(56)$. A key clinical question is whether or not the stricture is mainly due to inflammatory luminal narrowing, or fixed fibrosis; and the length of the stricture. Inflammatory strictures may be reversible with medical therapy, whereas fixed fibrotic lesions often require a mechanical solution - either balloon dilatation for strictures of 
$<4-5 \mathrm{~cm}$, or surgery (Figure 6a and 6b). If dilatation is on the cards, it is important to inform the endoscopist of the distance between the stricture and the ileocaecal valve (for small bowel strictures), since it is rarely possible to reach further than $15 \mathrm{~cm}$ or so into the terminal ileum without performing double-balloon enteroscopy at a specialist centre.

Penetrating disease may manifest as a localised inflammatory mass (sometimes called a phlegmon if there is solid material but no fluid, although some authorities frown on the term (56)), abscesses, sinuses and fistulas. Where a conglomerate of small bowel loops fistulate to each other (the "asterisk", "starfish" or "octopus" appearance), surgery is virtually inevitable. This should not be unduly delayed, since the process typically progresses and recruits more loops, ultimately rendering the resection more challenging and extensive. CT, MRI and US depict fistulas with similar sensitivity (25). Abscesses (outwith the context of recent intervention) generally result from penetrating disease complicated by infection, and are often better-shown on MRI or CT than US, since the deep pelvis is less accessible and gas-filled collections may be confused with the colon (Figure 6c).

\section{Predicting Patient Trajectory}

As described above, the course of $C D$ is highly variable. Accordingly, there is great interest in identifying which imaging findings at diagnosis predict longer-term outcome - i.e. who is destined to develop aggressive disease. Initial inflammatory burden may be misleading, since some patients will have a severe flare but respond well to treatment (and so have a good prognosis), whereas others may have low-level but refractory disease and ultimately suffer worse outcomes (Figure 7). Some clinical factors are consistently associated with poorer prognosis (e.g. young age at diagnosis, cigarette smoking, presence of perianal disease). The overall disease phenotype is also important - hospitalisation, surgery and debilitating symptoms are more common in patients with stricturing or penetrating disease (Montreal B2/B3) since, by their nature, these often require a surgical 
solution. Importantly, MRI seems to be superior to colonoscopy in predicting which patients are destined for worse outcomes; in one series (57), MR-diagnosed stenoses or intraabdominal fistulae were associated with future need for CD-related surgery, whereas colonoscopic findings were not. Determining which combination of clinical, endoscopic, biochemical and imaging parameters best predict which patients will develop "bad disease" (so needing more aggressive treatment) is an area of active research (Figure 8).

\section{Pre-operative Mapping}

Despite best efforts, ultimately some patients will require a surgical solution for their disease. This should not be viewed as a failure - for many, surgery is excellent treatment and leads to years of drug-free disease control. Once surgery is being considered, it is critical to arm the surgeon and patient with as much information as possible to ensure optimal decision-making. Radiologists must describe the number, length, and location of strictures and/or fistulas in relation to the ligament of Treitz, and indicate the presence of upstream dilatation(56). The length of bowel involved can dictate the surgical management, particularly if a resection will result in a short bowel; stricturoplasty may be necessary to preserve bowel length.

Proximal disease is important to detect, as it may prove necessary to defunction above a fistula repair (particularly if there is co-existent sepsis). This is simpler where the upstream stoma is not too proximal, since high jejunostomies do not permit sufficient incircuit bowel to sustain nutritional requirements, and may have torrential fluid losses. These cases favour judicious use of CTE and MRE, since enteric contrast permits accurate assessment of complex fistulating disease, differentiating enterocutaneous from enteroenteric tracts. Since luminal fluid and oral contrast for MRI are both T2 hyperintense, CTE (using positive iodinated contrast) may be the test of choice despite the radiation penalty. 
Off-label use of 1:100 dilute gadolinium, instilled via a stoma, fistula or per-rectum, can be considered in selected circumstances (Figure 9).

Although US has been shown to accurately map small bowel strictures(58), it is typically easier to communicate complex findings to the surgical team using anatomical reference points on images with which they are familiar, which naturally suits MRI and CT. Ultrasound enthusiasts may find hand-drawn diagrams to be a reasonable alternative.

\section{Post-operative Recurrence}

Post-resection, around $20 \%$ of patients will develop recurrent CD in the first postoperative year, with an $\sim 10 \%$ annual risk thereafter (59). Since endoscopic recurrence at 1 year predicts clinical recurrence within 5 years (60), active surveillance is advised (61-64). However, regular colonoscopy is costly and unpleasant for patients; therefore, non-invasive methods, including imaging, may also have a role (19). Yung et al reported that MRE and capsule endoscopy had sensitivities of $97 \%$ and $100 \%$, and specificities of $84 \%$ and $69 \%$ respectively, for detecting endoscopic recurrence(65). Similarly, SICUS was $97.5 \%$ accurate for recurrence at 1 year (60). US is intuitively attractive, since both the clinical question and anatomical focus of the examination are highly targeted, meaning a decision can be made rapidly and accurately. Accordingly, many centres will use imaging surveillance for recurrence in low-risk or asymptomatic patients, reserving colonoscopy for those at higher risk on clinical grounds, ongoing symptoms or with abnormal biomarkers.

\section{Developments \& Controversies}

\section{Use of contrast with ultrasonography}

SICUS uses oral contrast to improve visualisation of the bowel wall, separate adjacent loops and outline strictures and fistulas. SICUS may improve diagnostic sensitivity, 
although the incremental benefit over conventional US is uncertain. Certainly in the right hands it correlates well with both magnetic resonance enterography (MRE) and intraoperative findings for complicated disease (58). On the other hand, it is a comparatively more laborious, time-consuming technique and suffers from greater interobserver variability than conventional ultrasonography, limiting widespread adoption (66). Moreover, the need to ingest large volumes of oral contrast removes one of ultrasound's key advantages-"the drink" is the most disliked aspect of MR (24).

Contrast enhanced ultrasonography (CEUS) permits estimation of bowel wall vascularisation using intravenous injection of echobright microscopic bubbles. CEUS findings correlate with both inflammatory markers and clinical severity $(67,68)$ with a sensitivity and specificity of $93 \%$ and $87 \%$, respectively, in detecting active CD (69). CEUS also shows some promise in differentiating fibrotic disease from inflammation (70-72), although reports conflict (73). Further applications of CEUS include identifying treatment responders (74-77) and early detection of post-operative disease recurrence (78). There is some work suggesting that CEUS is additive to conventional US, by reducing indeterminate results while simultaneously improving activity assessment (67). Work is now concentrating on standardising acquisition parameters and post-processing software for CEUS; presently, results from one scanner/probe combination do not match those from different systems (79).

\section{Diffusion weighted imaging DWI}

Diffusion weighted imaging (DWI) relies on reduced molecular motion of water in inflamed bowel wall, causing high signal on high b-value images (typically $600-1000 \mathrm{~s} / \mathrm{mm}^{2}$ ). Although DWI helps detect disease, it cannot be used alone for characterisation, as fibrosis also causes restricted diffusion (80). Many investigators have tried to identify ADC values that discriminate actively inflamed from non-inflamed bowel; in our experience, this is challenging. Normal bowel is thin-walled and mobile, meaning ADC measurements often 
suffer partial volume averaging from adjacent luminal fluid (high ADC) or mesenteric fat (low $A D C)$. Moreover, fat deposition in the bowel wall spuriously lowers ADC measurements due to signal reduction on these inherently fat-suppressed sequences. We (81), and others (in a paediatric population (82)) found that ADC values had poor intra and inter-observer variability, whereas subjective assessment of DWI signal was useful to highlight areas of abnormality. Underlining this, a recent report showed that replacing IV contrast-enhanced sequences with DWI was non-inferior for detecting bowel inflammation (45). At our institution, we have largely replaced contrast-enhanced studies with DWI, reserving intravenous contrast for those with suspected fistulating disease and inpatients. This shortens the scan, reduces cost, and removes any risk of allergy or gadolinium deposition.

\section{Small bowel motility MRI}

Normal bowel wall motility is a complex neuromuscular function facilitated by luminal distension. MRI allows rapid, radiation-free motility assessment using fast T2-weighted SSFP or echo-planar sequences; 1 image frame/second for at least $15-20$ seconds is the recommended minimum (83). Normal gut shows smooth, progressive peristaltic waves from proximal to distal, and even underfilled segments of small bowel with normal peristalsis can be characterised as non-diseased. This is particularly helpful if enteric contrast medium has not reached the terminal ileum, or for segments of jejunal collapse - if they move normally, they are highly likely to be normal. As well as ruling out disease, cine sequences may rule it in - in one study of $40 \mathrm{CD}$ patients, motility sequences yielded 35 more CD-specific findings than conventional MRE alone (84).

Recent advances in post-processing software for small bowel motility imaging have enabled computerised quantification of motility, rather than visual assessment, which is subjective, time-consuming and non-reproducible. Semi-automated lumen calibre measurement and displacement mapping are the main software solutions available $(85,86)$. 
These not only allow disease detection, but also quantitation; terminal ileal motility scores are negatively correlated with histological disease activity (88) and show excellent agreement with the conventional morphological MaRIA score (87). Moreover, improved small bowel motility reflects treatment response; we found motility improvement post-therapy to be $93 \%$ sensitive and $77 \%$ specific for clinical response to anti-TNFa agents, a finding that is currently under prospective evaluation in the multicentre MOTILITY trial.

\section{Service configuration and delivery}

Setting up an MRE service is relatively straightforward, requiring training of MRI staff and local protocols to standardise patient preparation and the use of contrast. If enteroclysis is a consideration, facilities for fluoroscopic placement of naso-jejunal tubes should be available, although in practice the test is loathed by patients and rarely needed - where there is doubt, capsule endoscopy is a better alternative. MR lists can be run independently by radiographers at any time of the day, and the radiologist can report these studies when convenient. CTE can be used in those patients with contraindications to MRI and run in a similar fashion.

A SBUS service is more efficient in terms of increasing patient throughput and easing the burden on the MR scanner. However, it is complicated by the requirement to have a trained radiologist or sonographer on site to undertake the examinations, and robust cover is needed before deployment in the acute setting. This can be challenging, particularly for smaller units, as there is currently a paucity of practitioners with SBUS expertise.

\section{Conclusions}

The role of imaging in CD has expanded greatly in recent years and is now integral to diagnosis and management. US and MRE are at the forefront of the diagnostic pathway. The 
goal is for comprehensive imaging services to be delivered in an effective, cost efficient manner throughout the NHS. In the research arena, functional techniques such as DWI and motility imaging are providing new ways to quantify disease activity, monitor and even predict therapeutic response, with the goal of providing more personalised therapy. 


\section{Figure legends}

\section{Figure 1}

Ultrasound and MRI performed at initial diagnosis of Crohn's disease. The top row shows a case in which MRI missed proximal disease. Panel (a) shows a small bowel ultrasound of the terminal ileum with mural (arrow) and submucosal (arrowhead) thickening in keeping with active terminal ileal disease. The corresponding MRE (coronal HASTE) sequence in panel (b) shows a sub-optimally distended jejunum, prospectively reported as normal. Contemporaneous ultrasound in panel (c) depicts active jejunal disease (arrow), missed by MRI due to interpretive error. The bottom row shows the reverse situation, with US missing upstream disease well depicted by MRI. Panel (d) shows terminal ileal high T2 signal (arrowhead), mural thickening (arrow) and vascular engorgement (asterisk) on T2 fat suppressed MRI, with corresponding abnormality on same-day ultrasound in panel (e). However, ultrasound failed to detect the proximal ileal skip lesions shown on MRI, arrows in panel (f).

\section{Figure 2}

Typical patchy appearance of Crohn's disease. Axial T2 HASTE MRI (a) showing heterogenous mural thickening and patchy oedema of the terminal ileum, with some areas of moderately increased signal (arrow) and other areas of extremely bright signal (arrowhead) reflecting the within-slice variability of the disease. Features of asymmetry and ulceration are demonstrated. US shows loss of folds (arrow in b) with blurring and irregularity of the interface between the mesenteric border of the bowel and the adjacent mesentery. The mesentery itself is expanded towards the anti-mesenteric border in (arrows in c), the classical "creeping fat". The patchy nature of the disease in axial section is also apparent (asterisks). Coronal T2 HASTE MRI in (d) shows the longitudinal heterogeneity of the disease, with skip lesions (arrow) and variations in the degree of mural oedema (asterisks).

\section{Figure 3}


(a) Coronal HASTE - Inactive disease. High signal mural thickening within the terminal ileum may be due to fluid or fat. (b) Coronal HASTE with fat saturation shows signal suppression within the wall, denoting inactive disease with fat deposition. Active disease shows high signal mural thickening (greater intensity than surrounding muscle) both with and without fat suppression, in keeping with mural oedema.

\section{Figure 4}

(a) - (c) Mild, moderate and severe disease on US, with gradual increase in mural, mucosal and submucosal thickening, and vascularity on Doppler signal imaging. In mild disease, the mucosa may be thickened despite a normal $(<3 \mathrm{~mm})$ overall wall thickness (calipers in (a)). Similarly, mild, moderate and severe disease on MRI; (d) Mild disease on coronal HASTE with fat saturation - terminal ileum thickening and oedema. (e) Moderate disease on axial HASTE with fat saturation. Mucosal ulcerations (arrows) and mural thickening can be seen.

(f) Severe disease affecting multiple distal and proximal small bowel loops on coronal HASTE with fat saturation.

\section{$\underline{\text { Figure } 5}$}

Initial active disease on SBUS in panel (a), which responded to treatment with a reduction in mural thickening in panel (b). With a further 9 months of treatment, the US returned to normal (c), indicating imaging remission. In a different patient monitored via MRI, axial HASTE with fat saturation in (a) shows moderately active terminal ileal disease. The corresponding post treatment image in (e) shows only mild mural thickening and no oedema, in keeping with remission.

\section{Figure 6}

(a) Coronal HASTE with fat saturation. Terminal ileal stricture (asterisk) with mild upstream bowel dilatation and mural oedema in keeping with an inflammatory stricture. (b). Multiple strictures with intervening bowel dilatation. The combination of low T2 signal and enteric dilatation implies a fibrostenotic stricture. Ultrasound of the pelvic ileum (c) in a different 
patient shows thickened terminal ileum (arrows) and a posterior gas-filled structure, thought to represent the rectum (asterisk). Axial HASTE MR performed the following day (d) shows this to be a deep pelvic abscess (arrows).

\section{Figure 7}

Lemann Score for two patients. The Lemann score assess global gut damage and takes into account clinical events, imaging evaluation and endoscopic findings to represent bowel damage. Patient (a) demonstrates progressively complicating disease (red line) despite experiencing relatively mild, intermittent flare ups (black line). Patient (b), although presenting initially with severely active disease, due to good long-term control, accumulates less total bowel damage.

\section{Figure 8}

Examples of bad prognostic disease (a) coronal HASTE - a fibrotic stricture at the terminal ileum in a symptomatic patient with abdominal pain shows upstream bowel dilatation. The patient declined surgery, and despite maximal medical therapy, this progressed with further small bowel dilatation and loss of motile function in (b). Fibrotic stricturing disease with enteric dilatation strongly indicates surgery or dilatation.

\section{Figure 9}

Axial HASTE MR in (a) shows a fluid collection close to the top of a rectal stump (arrow) in a patient with complex pelvic Crohn's disease and a right iliac fossa ileal conduit (asterisk). The stump was originally stapled closed at the original surgical procedure. Dilute $(1: 100)$ gadolinium contrast instilled per rectum outlines the narrowed rectosigmoid in (b) with deep ulceration (arrows), and confirmed an enterocutaneous fistula via the subcutaneous collection (arrows in c) due to a "blown" rectal stump, which has essentially converted itself to a mucus fistula. 
Table 1. Typical MRI sequences used at our institution on a Siemens $1.5 \mathrm{~T}$ scanning unit.

\begin{tabular}{|c|c|c|c|c|c|c|c|c|}
\hline & & & & & VRI Sequence & & & \\
\hline & $\begin{array}{l}\text { T2 } \\
\text { TRUFI } \\
\text { COR }\end{array}$ & $\begin{array}{l}\text { T2 } \\
\text { TRUFI } \\
\text { TRA }\end{array}$ & $\begin{array}{l}\text { T2 TRUFI } \\
\text { motility }\end{array}$ & $\begin{array}{l}\text { T2 HASTE } \\
\text { COR (with } \\
\text { and without } \\
\text { FS) }\end{array}$ & $\begin{array}{l}\text { T2 HASTE } \\
\text { TRA ( with } \\
\text { and without } \\
\text { FS) }\end{array}$ & $\begin{array}{l}\text { DWI } \\
\text { TRA }\end{array}$ & $\begin{array}{l}\text { T1 VIBE +FS } \\
\text { COR (pre + post } \\
\text { contrast) }\end{array}$ & $\begin{array}{l}\text { T1 VIBE + FS } \\
\text { TRA }\end{array}$ \\
\hline FOV read (mm) & 400 & 350 & 420 & 400 & 360 & 351 & 400 & 380 \\
\hline FOV phase (\%) & 325 & 263 & 342 & 263 & 263 & 286 & 323 & 285 \\
\hline No. of slices & 25 & 34 & 1 & 24 & 34 & 18 & 40 & 72 \\
\hline Stacks & 1 & 2 & $\begin{array}{l}20 \\
\text { measures }\end{array}$ & 1 & 2 & 3 & 1 & 2 \\
\hline TR (ms) & 3.99 & 4.25 & 3.89 & 800 & 305 & 2500 & 3.24 & 4.9 \\
\hline TE (ms) & 1.72 & 2.13 & 1.95 & 86 & 86 & 85 & 1.24 & 2039 \\
\hline Image matrix & $166 \times 256$ & $150 \times 256$ & $162 \times 256$ & $167 \times 256$ & $134 \times 256$ & $156 \times 192$ & $176 \times 288$ & $163 \times 320$ \\
\hline $\begin{array}{l}\text { Slice thickness } \\
(\mathrm{mm})\end{array}$ & 4 & 4 & 10 & 4 & 4 & 5 & 3.5 & 4.9 \\
\hline Slice gap (\%) & 20 & 20 & 0 & 20 & 20 & 30 & 0 & 0 \\
\hline Signal averages & 1 & 1 & 1 & 1 & 1 & 3 & 1 & 1 \\
\hline iPAT & NA & NA & NA & GRAPPA $\times 2$ & GRAPPA x2 & $\begin{array}{l}\text { GRAPPA } \\
x 2\end{array}$ & GRAPPA $\times 2$ & GRAPPA x2 \\
\hline $\begin{array}{l}\text { b values } \\
\text { (s/mm2) }\end{array}$ & NA & NA & NA & NA & NA & 0,600 & NA & NA \\
\hline Flip angle & 46 & 61 & 64 & 150 & 150 & & 10 & 10 \\
\hline
\end{tabular}

ADC - Apparent diffusion co-efficient, BTFE - Balanced Turbo Field Echo, COR - coronal, DWI - Diffusion Weighted Imaging, FOV - Field of view FS - fat saturation, GAD gadolinium, GRAPPA - GeneRalized Autocalibrating Partial Parallel Acquisition, HASTE half-Fourier acquired single turbo spin-echo, IPAT - Integrated Parallel Acquisition Techniques, NA - not applicable, TE - time to echo, TR - time to repeat, TRA - transaxial, 
Trufi - True Fast Imaging with steady state precession, VIBE - volumetric interpolated breath-hold examination

Table 2. Scoring Method for the Magnetic Enterography Global Score (MEGS). Score each segment (jejunum, ileum, terminal ileum, caecum, ascending, transverse, descending, sigmoid colon and rectum) and then multiply each segmental score depending on the length of diseased segment. The terminal ileum is defined as the last $10 \mathrm{~cm}$ from the ileocaecal valve or ileocolic anastomosis. Thereafter, add 5 points for the presence of each of enlarged lymph nodes, comb sign, abscess or fistula are present (up to 20 additional points). Adapted from (41)

\begin{tabular}{|c|c|c|c|c|}
\hline Score & 0 & 1 & 2 & 3 \\
\hline $\begin{array}{l}\text { Mural thickness of small } \\
\text { bowel }^{*}\end{array}$ & $<3 \mathrm{~mm}$ & $>3-5 \mathrm{~mm}$ & $>5-7 \mathrm{~mm}$ & $>7 \mathrm{~mm}$ \\
\hline Mural T2 signal** & $\begin{array}{l}\text { Equivalent to } \\
\text { normal bowel wall }\end{array}$ & $\begin{array}{l}\text { Minor increase in } \\
\text { signal: bowel wall is } \\
\text { dark grey on fat- } \\
\text { saturated images }\end{array}$ & $\begin{array}{l}\text { Moderate increase in } \\
\text { signal: bowel wall is } \\
\text { light grey on fat- } \\
\text { saturated images }\end{array}$ & $\begin{array}{l}\text { Marked increase in } \\
\text { signal: bowel wall } \\
\text { has areas of white } \\
\text { high signal } \\
\text { approaching that of }\end{array}$ \\
\hline
\end{tabular}




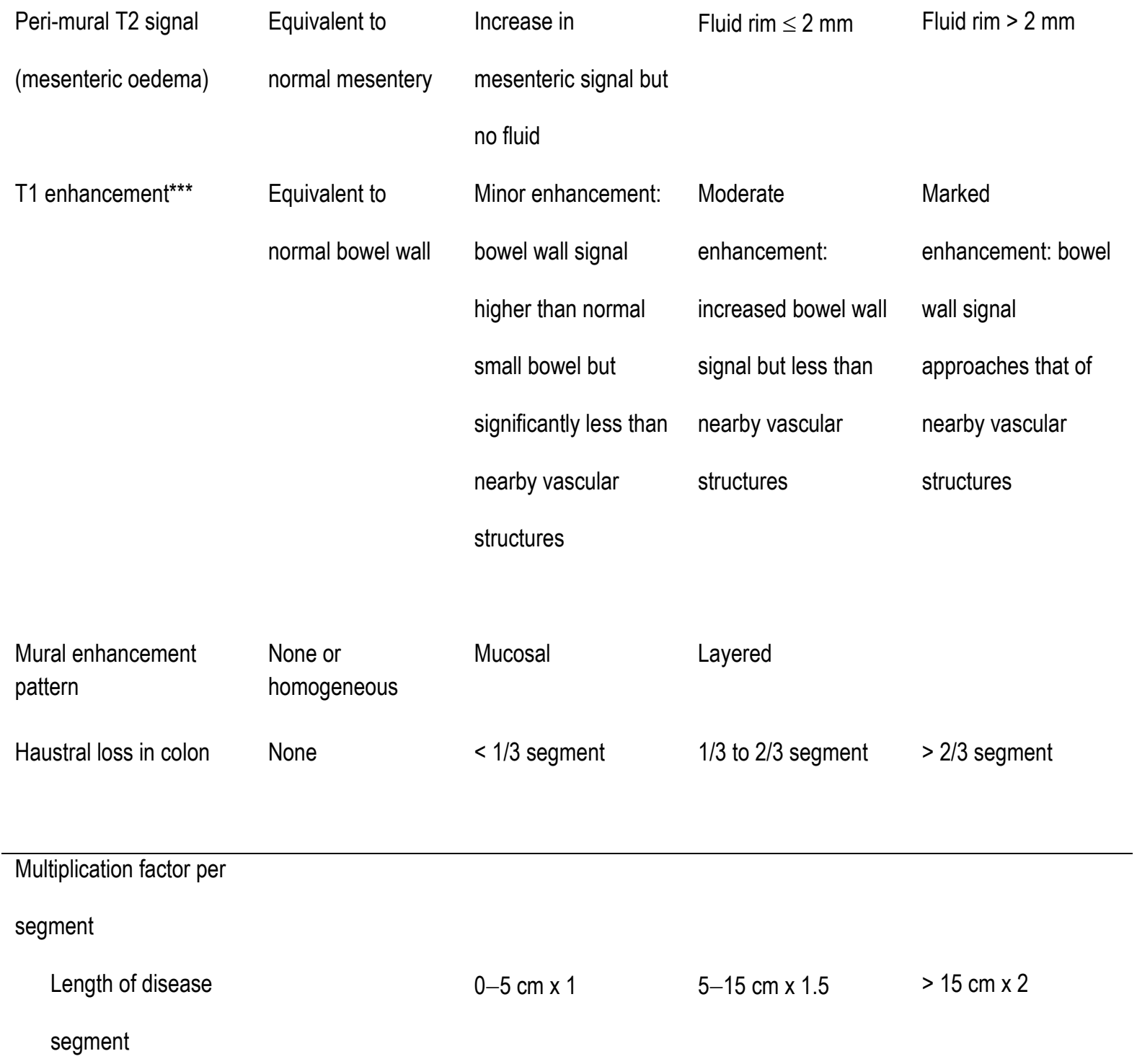

Additional score for extramural features:

$\begin{array}{lll}\text { Score } & 0 & 5 \\ \text { Lymph nodes } & \text { Absent } & \text { Present } \\ (\geq 1 \mathrm{~cm} \text { in shortest } & & \\ \text { diameter }) & \text { Absent } & \text { Present } \\ \text { Comb sign }{ }^{\Psi} & \text { Absent } & \text { Present } \\ \text { Abscess } & \text { Absent } & \text { Present }\end{array}$

Fistulae 
${ }^{*}$ Measured by electronic calipers, ${ }^{* *}$ compared with normal small bowel, ${ }^{* * *}$ compared with nearest vessel, ${ }^{\Psi}$ linear densities on the

mesenteric side of affected bowel segments

\section{References}

1. Carter MJ, Lobo AJ, Travis SP, Ibd Section BSoG. Guidelines for the management of inflammatory bowel disease in adults. Gut. 2004;53 Suppl 5:V1-16.

2. Van Assche G, Dignass A, Panes J, et al. The second European evidence-based Consensus on the diagnosis and management of Crohn's disease: Definitions and diagnosis. J Crohns Colitis.

2010;4(1):7-27.

3. Colombel JF, Sandborn WJ, Reinisch W, et al. Infliximab, azathioprine, or combination therapy for Crohn's disease. N Engl J Med. 2010;362(15):1383-1395.

4. D'Haens G, Baert F, van Assche G, et al. Early combined immunosuppression or conventional management in patients with newly diagnosed Crohn's disease: an open randomised trial. Lancet. 2008;371(9613):660-667.

5. Colombel JF, Panaccione R, Bossuyt $P$, et al. Effect of tight control management on Crohn's disease (CALM): a multicentre, randomised, controlled phase 3 trial. Lancet. 2018;390(10114):27792789.

6. Taylor SA, Avni F, Cronin CG, et al. The first joint ESGAR/ ESPR consensus statement on the technical performance of cross-sectional small bowel and colonic imaging. Eur Radiol. 2017;27(6):2570-2582.

7. Hafeez R, Greenhalgh R, Rajan J, et al. Use of small bowel imaging for the diagnosis and staging of Crohn's disease--a survey of current UK practice. Br J Radiol. 2011;84(1002):508-517.

8. Sahiner B, Chan H-P, Hadjiiski LM, et al. Multi-modality CADx: ROC Study of the Effect on Radiologists' Accuracy in Characterizing Breast Masses on Mammograms and 3D Ultrasound Images. ACADEMIC RADIOLOGY. 2009;16(7):810-818.

9. Hara AK, Leighton JA, Heigh RI, et al. Crohn disease of the small bowel: preliminary comparison among CT enterography, capsule endoscopy, small-bowel follow-through, and ileoscopy. Radiology. 2006;238(1):128-134.

10.Feuerbach S. MRI enterography: the future of small bowel diagnostics? Dig Dis. 2010;28(3):433438.

11.Kuehle CA, Ajaj W, Ladd SC, Massing S, Barkhausen J, Lauenstein TC. Hydro-MRI of the small bowel: effect of contrast volume, timing of contrast administration, and data acquisition on bowel distention. AJR Am J Roentgenol. 2006;187(4):W375-385.

12.Siddiki HA, Fidler JL, Fletcher JG, et al. Prospective comparison of state-of-the-art MR enterography and CT enterography in small-bowel Crohn's disease. AJR Am J Roentgenol. 2009;193(1):113-121.

13.Low RN, Francis IR, Politoske D, Bennett M. Crohn's disease evaluation: comparison of contrastenhanced MR imaging and single-phase helical CT scanning. J Magn Reson Imaging. 2000;11(2):127135.

14.Furukawa A, Saotome T, Yamasaki M, et al. Cross-sectional imaging in Crohn disease. Radiographics. 2004;24(3):689-702. 
15.Rubesin SE, Levine MS. Radiologic diagnosis of gastrointestinal perforation. Radiol Clin North Am. 2003;41(6):1095-1115, v.

16.Zissin R, Osadchy A, Gayer G. Abdominal CT findings in small bowel perforation. Br J Radiol. 2009;82(974):162-171.

17.Desmond AN, O'Regan K, Curran C, et al. Crohn's disease: factors associated with exposure to high levels of diagnostic radiation. Gut. 2008;57(11):1524-1529.

18.Sturm A, Maaser C, Calabrese E, et al. ECCO-ESGAR Guideline for Diagnostic Assessment in Inflammatory Bowel Disease. J Crohns Colitis. 2018.

19.Panes J, Bouhnik Y, Reinisch W, et al. Imaging techniques for assessment of inflammatory bowel disease: joint ECCO and ESGAR evidence-based consensus guidelines. J Crohns Colitis. 2013;7(7):556585.

20.Strobel D, Goertz RS, Bernatik T. Diagnostics in inflammatory bowel disease: ultrasound. World J Gastroenterol. 2011;17(27):3192-3197.

21.Fraquelli M, Sarno A, Girelli C, et al. Reproducibility of bowel ultrasonography in the evaluation of Crohn's disease. Dig Liver Dis. 2008;40(11):860-866.

22. Marteau P, Laharie D, Colombel JF, et al. Interobserver Variation Study of the Rutgeerts Score to Assess Endoscopic Recurrence after Surgery for Crohn's Disease. J Crohns Colitis. 2016;10(9):10011005.

23.Jairath V, Ordas I, Zou G, et al. Reliability of Measuring Ileo-Colonic Disease Activity in Crohn's Disease by Magnetic Resonance Enterography. Inflamm Bowel Dis. 2018;24(2):440-449.

24.Miles A, Bhatnagar G, Halligan S, et al. Magnetic resonance enterography, small bowel ultrasound and colonoscopy to diagnose and stage Crohn's disease: patient acceptability and perceived burden. Eur Radiol. 2018.

25.Panes J, Bouzas R, Chaparro M, et al. Systematic review: the use of ultrasonography, computed tomography and magnetic resonance imaging for the diagnosis, assessment of activity and abdominal complications of Crohn's disease. Aliment Pharmacol Ther. 2011;34(2):125-145.

26. Horsthuis K, Bipat S, Bennink RJ, Stoker J. Inflammatory bowel disease diagnosed with US, MR, scintigraphy, and CT: meta-analysis of prospective studies. Radiology. 2008;247(1):64-79.

27.Taylor SA, Mallett S, Bhatnagar G, et al. Diagnostic accuracy of magnetic resonance enterography and small bowel ultrasound for the extent and activity of newly diagnosed and relapsed Crohn's disease (METRIC): a multicentre trial. Lancet Gastroenterol Hepatol. 2018.

28. Mosli MH, Zou G, Garg SK, et al. C-Reactive Protein, Fecal Calprotectin, and Stool Lactoferrin for Detection of Endoscopic Activity in Symptomatic Inflammatory Bowel Disease Patients: A Systematic Review and Meta-Analysis. Am J Gastroenterol. 2015;110(6):802-819; quiz 820.

29. Barber JL, Maclachlan J, Planche K, et al. There is good agreement between MR enterography and bowel ultrasound with regards to disease location and activity in paediatric inflammatory bowel disease. Clin Radiol. 2017;72(7):590-597.

30.Triester SL, Leighton JA, Leontiadis GI, et al. A meta-analysis of the yield of capsule endoscopy compared to other diagnostic modalities in patients with non-stricturing small bowel Crohn's disease. Am J Gastroenterol. 2006;101(5):954-964.

31.Satsangi J, Silverberg MS, Vermeire S, Colombel JF. The Montreal classification of inflammatory bowel disease: controversies, consensus, and implications. Gut. 2006;55(6):749-753.

32.Park SH. DWI at MR Enterography for Evaluating Bowel Inflammation in Crohn Disease. AJR Am J Roentgenol. 2016;207(1):40-48.

33.Rimola J, Alvarez-Cofino A, Perez-Jeldres T, et al. Increasing efficiency of MRE for diagnosis of Crohn's disease activity through proper sequence selection: a practical approach for clinical trials. Abdom Radiol (NY). 2017;42(12):2783-2791.

34.Bhatnagar G, Von Stempel C, Halligan S, Taylor SA. Utility of MR enterography and ultrasound for the investigation of small bowel Crohn's disease. J Magn Reson Imaging. 2017;45(6):1573-1588.

35.Rimola J, Rodriguez S, García-Bosch O, et al. Magnetic resonance for assessment of disease activity and severity in ileocolonic Crohn's disease. Gut. 2009;58(8):1113-1120. 
36.Steward MJ, Punwani S, Proctor I, et al. Non-perforating small bowel Crohn's disease assessed by MRI enterography: derivation and histopathological validation of an MR-based activity index. Eur J Radiol. 2012;81(9):2080-2088.

37. Makanyanga JC, Pendse D, Dikaios N, et al. Evaluation of Crohn's disease activity: initial validation of a magnetic resonance enterography global score (MEGS) against faecal calprotectin. Eur Radiol. 2014;24(2):277-287.

38.Klang E, Amitai MM, Lahat A, et al. Capsule Endoscopy Validation of the Magnetic Enterography Global Score in Patients with Established Crohn's Disease. J Crohns Colitis. 2018;12(3):313-320. 39.Rimola J, Ordas I, Rodriguez S, et al. Magnetic resonance imaging for evaluation of Crohn's disease: validation of parameters of severity and quantitative index of activity. Inflamm Bowel Dis. 2011;17(8):1759-1768.

40.Taylor SA, Punwani S, Rodriguez-Justo M, et al. Mural Crohn disease: correlation of dynamic contrast-enhanced MR imaging findings with angiogenesis and inflammation at histologic examination--pilot study. Radiology. 2009;251(2):369-379.

41.Punwani S, Rodriguez-Justo M, Bainbridge A, et al. Mural inflammation in Crohn disease: locationmatched histologic validation of MR imaging features. Radiology. 2009;252(3):712-720.

42. Rimola J, Planell N, Rodriguez S, et al. Characterization of inflammation and fibrosis in Crohn's disease lesions by magnetic resonance imaging. Am J Gastroenterol. 2015;110(3):432-440.

43.Gulani V, Calamante F, Shellock FG, Kanal E, Reeder SB, International Society for Magnetic Resonance in $\mathrm{M}$. Gadolinium deposition in the brain: summary of evidence and recommendations. Lancet Neurol. 2017;16(7):564-570.

44.Buisson A, Joubert A, Montoriol PF, et al. Diffusion-weighted magnetic resonance imaging for detecting and assessing ileal inflammation in Crohn's disease. Aliment Pharmacol Ther.

2013;37(5):537-545.

45.Seo N, Park SH, Kim KJ, et al. MR Enterography for the Evaluation of Small-Bowel Inflammation in Crohn Disease by Using Diffusion-weighted Imaging without Intravenous Contrast Material: A Prospective Noninferiority Study. Radiology. 2016;278(3):762-772.

46. Kim JS, Jang HY, Park SH, et al. MR Enterography Assessment of Bowel Inflammation Severity in Crohn Disease Using the MR Index of Activity Score: Modifying Roles of DWI and Effects of Contrast Phases. AJR Am J Roentgenol. 2017;208(5):1022-1029.

47.Drews BH, Barth TF, Hanle MM, et al. Comparison of sonographically measured bowel wall vascularity, histology, and disease activity in Crohn's disease. Eur Radiol. 2009;19(6):1379-1386. 48.Bots S, Nylund K, Lowenberg M, Gecse K, Gilja OH, D'Haens G. Ultrasound for Assessing Disease Activity in IBD Patients: A Systematic Review of Activity Scores. J Crohns Colitis. 2018;12(8):920-929. 49. Novak KL, Kaplan GG, Panaccione R, et al. A Simple Ultrasound Score for the Accurate Detection of Inflammatory Activity in Crohn's Disease. Inflamm Bowel Dis. 2017;23(11):2001-2010.

50.Peyrin-Biroulet L, Sandborn W, Sands BE, et al. Selecting Therapeutic Targets in Inflammatory Bowel Disease (STRIDE): Determining Therapeutic Goals for Treat-to-Target. Am J Gastroenterol. 2015;110(9):1324-1338.

51.Deepak P, Fletcher JG, Fidler JL, et al. Radiological Response Is Associated With Better Long-Term Outcomes and Is a Potential Treatment Target in Patients With Small Bowel Crohn's Disease. Am J Gastroenterol. 2016;111(7):997-1006.

52.De Cruz P, Kamm MA, Prideaux L, Allen PB, Moore G. Mucosal healing in Crohn's disease: a systematic review. Inflamm Bowel Dis. 2013;19(2):429-444.

53.Vuitton L, Marteau P, Sandborn WJ, et al. IOIBD technical review on endoscopic indices for Crohn's disease clinical trials. Gut. 2016;65(9):1447-1455.

54.Pariente B, Cosnes J, Danese S, et al. Development of the Crohn's disease digestive damage score, the Lémann score. Inflamm Bowel Dis. 2011;17(6):1415-1422.

55.Pariente B, Mary JY, Danese S, et al. Development of the Lemann index to assess digestive tract damage in patients with Crohn's disease. Gastroenterology. 2015;148(1):52-63 e53. 
56.Bruining DH, Zimmermann EM, Loftus EV, Jr., et al. Consensus Recommendations for Evaluation, Interpretation, and Utilization of Computed Tomography and Magnetic Resonance Enterography in Patients With Small Bowel Crohn's Disease. Radiology. 2018;286(3):776-799.

57.Jauregui-Amezaga A, Rimola J, Ordas I, et al. Value of endoscopy and MRI for predicting intestinal surgery in patients with Crohn's disease in the era of biologics. Gut. 2015;64(9):1397-1402.

58. Kumar S, Hakim A, Alexakis C, et al. Small intestinal contrast ultrasonography for the detection of small bowel complications in Crohn's disease: correlation with intraoperative findings and magnetic resonance enterography. J Gastroenterol Hepatol. 2015;30(1):86-91.

59.Rutgeerts P. Protagonist: Crohn's disease recurrence can be prevented after ileal resection. Gut. 2002;51(2):152-153.

60.Onali S, Calabrese E, Petruzziello C, et al. Post-operative recurrence of Crohn's disease: A prospective study at 5 years. Dig Liver Dis. 2016;48(5):489-494.

61.Regueiro M, Schraut W, Baidoo L, et al. Infliximab prevents Crohn's disease recurrence after ileal resection. Gastroenterology. 2009;136(2):441-450.e441; quiz 716.

62.Sorrentino D, Terrosu G, Avellini C, Maiero S. Infliximab with low-dose methotrexate for prevention of postsurgical recurrence of ileocolonic Crohn disease. Arch Intern Med.

2007;167(16):1804-1807.

63. Regueiro M, Kip KE, Baidoo L, Swoger JM, Schraut W. Postoperative therapy with infliximab prevents long-term Crohn's disease recurrence. Clin Gastroenterol Hepatol. 2014;12(9):14941502.e1491.

64.Ferrante M, Papamichael K, Duricova D, et al. Systematic versus Endoscopy-driven Treatment with Azathioprine to Prevent Postoperative Ileal Crohn's Disease Recurrence. J Crohns Colitis. 2015;9(8):617-624.

65.Yung DE, Har-Noy O, Tham YS, et al. Capsule Endoscopy, Magnetic Resonance Enterography, and Small Bowel Ultrasound for Evaluation of Postoperative Recurrence in Crohn's Disease: Systematic Review and Meta-Analysis. Inflamm Bowel Dis. 2017;24(1):93-100.

66. Mocci G, Migaleddu V, Cabras F, et al. SICUS and CEUS imaging in Crohn's disease: an update. J Ultrasound. 2017;20(1):1-9.

67.Medellin-Kowalewski A, Wilkens R, Wilson A, Ruan J, Wilson SR. Quantitative Contrast-Enhanced Ultrasound Parameters in Crohn Disease: Their Role in Disease Activity Determination With Ultrasound. AJR American journal of roentgenology. 2016;206(1):64-73.

68.Saevik F, Nylund K, Hausken T, Odegaard S, Gilja OH. Bowel perfusion measured with dynamic contrast-enhanced ultrasound predicts treatment outcome in patients with Crohn's disease. Inflammatory bowel diseases. 2014;20(11):2029-2037.

69. Ma X, Li Y, Jia H, et al. Contrast-enhanced ultrasound in the diagnosis of patients suspected of having active Crohn's disease: meta-analysis. Ultrasound in medicine \& biology. 2015;41(3):659-668. 70.Ripolles T, Rausell N, Paredes JM, Grau E, Martinez MJ, Vizuete J. Effectiveness of contrastenhanced ultrasound for characterisation of intestinal inflammation in Crohn's disease: a comparison with surgical histopathology analysis. Journal of Crohn's \& colitis. 2013;7(2):120-128. 71.Quaia E, De Paoli L, Stocca T, Cabibbo B, Casagrande F, Cova MA. The value of small bowel wall contrast enhancement after sulfur hexafluoride-filled microbubble injection to differentiate inflammatory from fibrotic strictures in patients with Crohn's disease. Ultrasound in medicine \& biology. 2012;38(8):1324-1332.

72.Nylund K, Jirik R, Mezl M, et al. Quantitative contrast-enhanced ultrasound comparison between inflammatory and fibrotic lesions in patients with Crohn's disease. Ultrasound in medicine \& biology. 2013;39(7):1197-1206.

73. Wilkens R, Hagemann-Madsen RH, Peters DA, et al. Validity of Contrast-enhanced Ultrasonography and Dynamic Contrast-enhanced MR Enterography in the Assessment of Transmural Activity and Fibrosis in Crohn's Disease. J Crohns Colitis. 2018;12(1):48-56. 
74. Moreno N, Ripolles T, Paredes JM, et al. Usefulness of abdominal ultrasonography in the analysis of endoscopic activity in patients with Crohn's disease: changes following treatment with immunomodulators and/or anti-TNF antibodies. J Crohns Colitis. 2014;8(9):1079-1087.

75. Quaia E, Cabibbo B, De Paoli L, Toscano W, Poillucci G, Cova MA. The value of time-intensity curves obtained after microbubble contrast agent injection to discriminate responders from nonresponders to anti-inflammatory medication among patients with Crohn's disease. European radiology. 2013;23(6):1650-1659.

76.Quaia E, Migaleddu V, Baratella E, et al. The diagnostic value of small bowel wall vascularity after sulfur hexafluoride-filled microbubble injection in patients with Crohn's disease. Correlation with the therapeutic effectiveness of specific anti-inflammatory treatment. European journal of radiology. 2009;69(3):438-444.

77.Quaia E, Sozzi M, Angileri R, Gennari AG, Cova MA. Time-Intensity Curves Obtained after Microbubble Injection Can Be Used to Differentiate Responders from Nonresponders among Patients with Clinically Active Crohn Disease after 6 Weeks of Pharmacologic Treatment. Radiology. 2016;281(2):606-616.

78.Paredes JM, Ripolles T, Cortes X, et al. Contrast-enhanced ultrasonography: usefulness in the assessment of postoperative recurrence of Crohn's disease. Journal of Crohn's \& colitis. 2013;7(3):192-201.

79.Zink F, Kratzer W, Schmidt S, et al. Comparison of Two High-End Ultrasound Systems for ContrastEnhanced Ultrasound Quantification of Mural Microvascularity in Crohn's Disease. Ultraschall Med. 2016;37(1):74-81.

80.Tielbeek JA, Ziech ML, Li Z, et al. Evaluation of conventional, dynamic contrast enhanced and diffusion weighted MRI for quantitative Crohn's disease assessment with histopathology of surgical specimens. Eur Radiol. 2014;24(3):619-629.

81.Pendse DA, Makanyanga JC, Plumb AA, et al. Diffusion-weighted imaging for evaluating inflammatory activity in Crohn's disease: comparison with histopathology, conventional MRI activity scores, and faecal calprotectin. Abdom Radiol (NY). 2017;42(1):115-123.

82. Watson T, Calder A, Barber JL. Quantitative bowel apparent diffusion coefficient measurements in children with inflammatory bowel disease are not reproducible. Clin Radiol. 2018;73(6):574-579. 83. de Jonge CS, Gollifer RM, Nederveen AJ, et al. Dynamic MRI for bowel motility imaging-how fast and how long? Br J Radiol. 2018:20170845.

84.Froehlich JM, Waldherr C, Stoupis C, Erturk SM, Patak MA. MR motility imaging in Crohn's disease improves lesion detection compared with standard MR imaging. European radiology.

2010;20(8):1945-1951.

85.Bickelhaupt S, Froehlich JM, Cattin R, et al. Software-assisted quantitative analysis of small bowel motility compared to manual measurements. Clinical radiology. 2014;69(4):363-371.

86.Odille F, Menys A, Ahmed A, Punwani S, Taylor SA, Atkinson D. Quantitative assessment of small bowel motility by nonrigid registration of dynamic MR images. Magnetic resonance in medicine: official journal of the Society of Magnetic Resonance in Medicine / Society of Magnetic Resonance in Medicine. 2012;68(3):783-793.

87. Menys A, Puylaert C, Tutein Nolthenius CE, et al. Quantified Terminal lleal Motility during MR Enterography as a Biomarker of Crohn Disease Activity: Prospective Multi-Institution Study. Radiology. 2018:180100. 